\section{AB1192 CLINICAL APPLICATION OF 18F-FDG PET/CT IN RHEUMATIC DISEASES}

Hua Zhong. PEKING UNIVERSITY PEOPLE'S HOSPITAL, DEPARTMENT OF RHEUMATOLOGY, BEIJING, China

Background: ${ }^{18} \mathrm{~F}$-Flurodeoxyglucose Positron Emission Tomography/Computed Tomography $\left({ }^{18} \mathrm{~F}-\mathrm{FDG} \mathrm{PET} / \mathrm{CT}\right)$ is widely used in diagosing malignant diseases. It is also useful applying to autoimmune diseases.

Objectives: To investigate the clinical application and significance of ${ }^{18} \mathrm{~F}$ Flurodeoxyglucose Positron Emission Tomography/Computed Tomography $\left({ }^{18} \mathrm{~F}\right.$-FDG PET/CT $)$ in rheumatic diseases.

Methods: Patients who underwent ${ }^{18} \mathrm{~F}-\mathrm{FDG}$ PET/CT in the Department of Rheumatology and Immunology, Peking University People's Hospital from January 2012 to July 2018 were retrospectively analyzed for clinical application and imaging characteristics of ${ }^{18} \mathrm{~F}-\mathrm{FDG}$ PET/CT in rheumatic diseases.

Results: 1. From 2012 to 2018, 459 patients underwent ${ }^{18}$ F-FDG PET/CT examination in the Department of Rheumatology and Immunology, accounting for $5.79 \%$ of the total number of PET/CT examinations in the hospital. Further analysis of 415 patients with complete data, including 158 males $(38.07 \%)$ and 257 females $(61.93 \%)$, male to female ratio of 1:1.6, average age of patients are $(57 \pm 16.2)$ years old. The highest proportion group of patients is from 45 to 65 years old (201/415, $48.43 \%$ ). The total number of PET-CT examinations in the whole hospital increased year by year. Compared with 2012, the number has increased nearly 10 times in 2017 among patients in the Department of Rheumatology and Immunology. 2. In patients with rheumatic diseases, major purpose of PET/CT examination included exclusion of tumors, diagnosis, and assessment of the disease activity. 3. Of all the 459 patients, 315 cases may indicate the differential diagnosis of the disease, of which 269 $(85.4 \%)$ were highly suggestive of rheumatic clinical diagnosis, including 55 cases of vasculitis (17.46\%), 54 cases of myositis (17.14\%), 34 cases of rheumatoid arthritis $(10.79 \%), 30$ cases of Sjogren's syndrome $(9.52 \%)$, 27 cases of adult still's disease (8.57\%), 25 cases of systemic lupus erythematosus $(7.94 \%), 25$ cases of lgG4-related diseases (7.94\%), 10 cases of rheumatic polymyalgia $(3.17 \%)$ and 6 cases of systemic sclerosis $(1.9 \%)$; the remaining 46 cases $(14.6 \%)$ only suggested the possibility of autoimmune diseases.

Conclusion: Rheumatic diseases are complex and diverse, and it is difficult to diagnose. The application of ${ }^{18} \mathrm{~F}$-FDG PET/CT in the diagnosis of diseases is increasingly widespread. The results of this study suggest that ${ }^{18} \mathrm{~F}$-FDG PET/CT to some extent has significance in the classification and diagnosis of rheumatic diseases, especially for the exclusion of malignant tumors.

Disclosure of Interests: None declared

DOI: 10.1136/annrheumdis-2019-eular.8333

\section{AB1192B DIFFUSE WEIGHT IMAGE IS A POTENTIAL MAGNETIC RESONANCE SEQUENCE IN THE PREDICTION OF SPINAL SYNDESMOPHYTE IN YOUNG PATIENT WITH ANKYLOISNG SPONDYLITIS}

Sang Yeob Lee, Won Tae Chung, Sung Won Lee, Jin Woo Jeong. Dong-A university, rheumatology, busan, Korea, Rep. of (South Korea)

Background: Diffusion-weighted imaging (DWI) is an MRI sequence and has been shown to have advantages over standard MRI sequences in some clinical settings, such as early diagnosis of ischemic stroke and in staging some type of cancers. Different measures of diffusion have been proposed, with the apparent diffusion coefficient (ADC) measure the most widely used. Severe Inflammation leads to higher ADC values through increased water in extracellular, less constrained, spaces. Several studies have investigated the clinical utility of DWI in AS, with suggestive evidence that this sequence has valuable discriminatory capacity between AS and non-inflammatory back pain by inflammation degree on sacroiliac joint (SIJ).
Table 1. Scatterplots of the correlation between ADC value by MR DWI and the radiographic spinal progression over 4 years

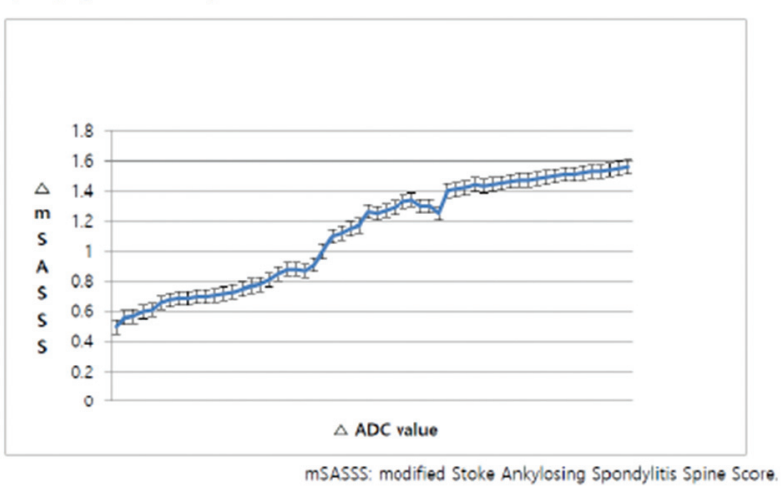

Table 2. The univariate and multivariate analysis of affecting factor for new spinal syndesmophyte

\begin{tabular}{l|c|c|c|c|}
\hline \multirow{2}{*}{ variate } & \multicolumn{2}{|l|}{ Univariated anylysis } & \multicolumn{2}{l}{ Multivariated analysis } \\
\cline { 2 - 5 } & OR (95\%) & p-ralue & \multicolumn{2}{l}{$O(95 \%)$} \\
\hline ADC value & $3.88(0.76-4.25)$ & 0.03 & $2.02(0.89-3.56)$ & 0.01 \\
\hline AS-DAS & $2.01(0.52-2.28)$ & 0.04 & $1.01(0.56-2.65)$ & 0.09 \\
\hline BASDAI & $0.89(0.78-1.02)$ & 0.03 & $0.67(0.34-1.78)$ & 0.08 \\
\hline BASMI & $0.58(0.52-1.22)$ & 0.09 & $0.88(0.59-1.88)$ & 0.34 \\
\hline BASFI & $0.31(0.11-0.68)$ & 0.13 & $0.34(0.12-0.78)$ & 0.12 \\
\hline
\end{tabular}

Table 1

Objectives: This study was tested the potential capacity of ADC value estimated by DWI on SIJ as the predictor of new spinal syndesmophyte in young patients with ankylosing spondylitis (AS).

Methods: The 58 patients who fulfilled the ASAS axSpA criteria were enrolled and their age was 18-23 years old. All subjects underwent MR on SIJ with oblique coronal images parallel to the long axis of the sacrum (fast spin-echo T1WI and STIR) at diagnosis and lumbar spine radiograph at diagnosis, 2 and 4 years sequentially. The ADC value on SIJ measured by DW-MRI at diagnosis and spinal radiographs were scored using the stoke AS Spinal Score (SASSS) at diagnosis, 2 and 4 years continuously. All patients were underwent a detailed clinical evaluation, including the serum erythrocyte sedimentation rate, C-reactive protein BASDAI, BASMI and BASFI. The univariate and multivariate logistic regression analysis was performed to identify that $A D C$ value on SIJ had an important influence on spinal syndesmophyte growth.

Results: The ADC value on SIJ showed a positive association with inflammatory marker such as, ESR and CRP, but no association with BASDAI, BASMI and BASFI. However, AS-DAS showed a positive association with $A D C$ value. The high ADC value showed more strongly associated with high SASSS over 2 and 4 years continuously. The univariate logistics analysis showed ADC value and AS-DAS were significant predic tors for new spinal syndesmophyte. However, multivariate logistics analysis showed the high ADC value had the highest risk of developing new syndesmophytes in spine.

Conclusion: This study showed that the inflammation of AS patient was a positive association with ADC value on SIJ by DW-MRI and the measured the ADC value on SIJ by DW-MRI was the modest discriminating capacity method for predicting development of new spinal syndesmophyte in young AS patients.

\section{REFERENCES}

[1] Sequence in the Diagnosis of Ankylosing Spondylitis Diffusion-weighted Imaging Is a Sensitive and Specific Magnetic Resonance Robinson, Nivene Saad, Kim-Anh Lê Cao and Matthew A. Brown Linda A. Bradbury, Kelly A. Hollis, Benoît Gautier, Sateesh Shankaranarayana, Philip C. J Rheumatol 2018;45;771-778 
Acknowledgement: None

Disclosure of Interests: None declared

DOI: 10.1136/annrheumdis-2019-eular.1876

\section{Public health, health services research, and health economics}

\section{AB1193 OBSERVATIONAL PROSPECTIVE COHORT STUDY TO EVALUATE EFFICACY AND SAFETY OF TAPENTADOL IN PATIENTS WITH RESPIRATORY DISEASE}

Maria Del Pilar Ahijado Guzman ${ }^{1}$, Raul Maria Veiga Cabello², Miguel Cantalejo Moreira ${ }^{1}$, Justo Ruiz Ruiz ${ }^{3}$, Antonio Zapatero Gaviria ${ }^{3}$. ${ }^{1}$ Htal Universitario de Fuenlabrada, Unit of Rheumatology, Fuenlabrada, Spain; ${ }^{2}$ Htal Universitario Central de la Defensa, Rheumatology Service, Madrid, Spain; ${ }^{3} \mathrm{Htal}$ Universitario de Fuenlabrada, Service of Internal Medicine, Fuenlabrada, Spain

Background: Chronic pain analgesia is a concern in clinical practice in rheumatic patients, especially when the intensity is severe. In this case opioids are indicated but also contraindicated in cases of important respiratory depression and, therefore, must be administered with caution to patients with respiratory disease (1). Many of our patients are not in the condition of severe respiratory depression although they present plurypathologies, which could be triggered at the use of certain doses (2). For this reason, it is important the existence of a study that shows that tapentadol is safe in pluripathological patients when used at regular doses in clinical daily practice in Reumathology (3).

Objectives: Single site, non-postmarketing observational study. The main objective is to evaluate the safety of tapentadol prolonged release (TPR) $50 \mathrm{mg} / 12 \mathrm{~h}$, measured as tolerance ("good", "bad" or "not too bad") and by the comparative analysis of gradients between both groups of the study (control group and pathological group), the basal oxygen saturation, and after the dose of TPR (basal pulse oximetry minus the mean of the oxygen saturation after 30 days of study).

Methods: Inclusions criteria arepatients with severe chronic pain (Visual Analogical Scale, VAS $>4$ ) diagnosed from mild to severe chronic obstructive pulmonary disease (COPD) (spirometry after bronchodilatation with forced expiratory volume (FEV)/forced vital capacity $(F V C)<70 \%$ and FEV1 $=50 \%$ ), and/or obesity, and/or controlled asthma and/or other conditions likely to produce respiratory depression with opioids (pathological group). Exclusion criteria consists in basal oxigen saturation measured by the pulse oximeter inferior to $92 \%$. A descriptive analysis of variables and a comparison of the means were performed.

Results: 29 patients; 12 in control group and 17 pathological group (obesity: 9, controlled asthma: 3; mild to moderate COPD: 7; other pathologies: 7). Overall, the type of pain was nociceptive $59 \%$, neuropathic $21 \%$ and mixed $20 \%$; mainly women $(67 \%)$, caucasic race $(92 \%)$, median age 60 years old, and with $93 \%$ good tolerability and $97 \%$ good treatment adherence. Concerning results per groups, at control group, VAS mean, arterial pressure (AP), oxygen saturation (SO2) and heart rate (HR) before and after treatment. 8,3 vs 5,$8 ; 127 / 74$ vs $124 / 73(\mathrm{mmHg}) ; 95,6$ vs $95,7(\%) ; 76$ vs 73 (bpm). In the pathological group: 7,5 vs 5,$6 ; 131 /$ 82 vs $127 / 78(\mathrm{mmHg}) ; 96$ vs 95,5 (\%); 75 vs 76 bpm. Regarding the results as per gradients between groups, no statistically significant differences were found, except for VAS, $(p=0,00008)$. There were no cases of decrease of the oxygen saturation below $92 \%$ along the study.

Conclusion: The results support the safety of tapentadol from the respiratory point of view, measured by oxygen saturation, since no statistically significant differences were found between both groups, and, due to the excellent tolerability, as no clinical data showed signs of hypercapnia. No statistically significant differences were found in the oxygen saturation between both groups with the intake of TPR, with excellent tolerability and treatment adherence. There were no cases of decrease of the oxygen saturation below $92 \%$ along the study.

\section{REFERENCES}

[1] Carr DB, How prevalent is chronic pain?. Pain clinical updates 2003; (11) 2:1-4

[2] Breivik H, ColletB, Ventafridda V, Cohen R, Gallacher, D. Survey of chronic pain in Europa: Prevalence, impact on daily life, and treatment. Eur J Pain 2006; 10 (4) 287-333

[3] Ficha Tecnica Palexia ${ }^{\circledR}$ Retard.

Disclosure of Interests: None declared DOI: 10.1136/annrheumdis-2019-eular.3220

\section{$\mathrm{AB} 1194$}

KNOWLEDGE AND PERCEPTIONS OF PORTUGUESE FAMILY PHYSICIANS TOWARDS ANKYLOSING SPONDYLITIS: RESULTS FROM THE ASSESSMENT OF RESULTS IN ANKYLOSING SPONDYLITIS(AREA) STUDY

Filipe Araújo ${ }^{1}$, Cláudia Duarte ${ }^{2}$, Jorge Brandão ${ }^{3}$, Rui Cernadas ${ }^{4}$, Elsa Mateus ${ }^{5}$ Luís Cunha Miranda ${ }^{6}$, José Canas Da Silva ${ }^{7}$, José Gomes Da Silva ${ }^{8}$, Pedro Simões Coelho ${ }^{2} .{ }^{1}$ Hospital de Sant'Ana, Parede, Portugal; ${ }^{2}$ NOVA IMS Information Management School, Lisboa, Portugal; ${ }^{3}$ Apmgf - Associação Portuguesa De Medicina Geral E Familiar, Lisboa, Portugal; ${ }^{4}$ Serviços Médicos Continental, Gaia, Portugal; ${ }^{5}$ Liga Portuguesa Contra as Doenças Reumáticas, Lisboa, Portugal; ${ }^{6}$ IPR - Portuguese Institute of Rheumatology, Lisboa, Portugal; ${ }^{7}$ Hospital Garcia de Orta, Almada, Portugal; ${ }^{8}$ National Association of Ankylosing Spondylitis, São Domingos de Rana, Portugal

Background: Ankylosing spondylitis (AS) patients have a significant delay between symptom onset and disease diagnosis, reaching on average 7 to 10 years in developed countries. Understanding the reasons behind this delay is essential to reduce the individual and socio-economic burden of the disease.

Objectives: To assess knowledge and perceptions of Portuguese family physicians (FP) towards AS and determine whether these contribute to the diagnostic delay at the primary care level.

Methods: The Assessment of REsults in Ankylosing spondylitis (arEA) study was developed by the NOVA-Information Management School (Lis bon) in collaboration with the Portuguese Society of Rheumatology, the Portuguese Association of Family Physicians (APMGF), the National Association of Primary Care Units (USF-AN), the National Association of AS Patients and the Portuguese League Against Rheumatic Diseases. The arEA aimed at assessing reasons for delayed diagnosis of $A S$, as well as disease impact in patients' lives, global health and work. A comprehensive online survey was developed and sent to FP associated with APMGF and USF-AN, collecting data on demographics, global knowledge and diagnostic and treatment attitudes towards AS.

Results: 91 FP responded the survey, $51.6 \%$ female, more frequently from the 25-44 year-old age group, half of which with $<5$ years of clinical experience. Most FP (70\%) did not consider AS to be a relevant dis ease in everyday clinical practice but recognized (90\%) there was a delay in diagnosis (5 years on average). Nevertheless, knowledge over AS was adequate. On average, prevalence was considered to be 56 cases per 1000 persons (close to the actual prevalence of 47 cases per 1000 persons reported in the epidemiological study EpiReumaPt). When assessing a patient with suspicious AS, the most valued symptoms/signs were inflammatory back/buttock pain, extra-articular manifestations (uveitis, enthesitis, dactylitis, psoriasis) and sacroiliitis on imaging (4.1, 3.9 and 3.9 on a $1-6$ scale, respectively); $92.5 \%$ of FP refer the patient to a hospital consultation, rheumatology in $88.5 \%$ of cases; $37.5 \%$ of FP initiate treatment, with NSAIDs in $81 \%$ of cases. A mean delay of 9 months between patient referral and first hospital consultation was also reported (>1 year in 22\%). In $73.4 \%$ of cases, no specific referral proto col exists for AS or other rheumatic inflammatory conditions; $33.8 \%$ of FP felt that the development of such protocol would improve access while $36.8 \%$ considered that a rheumatologist acting as consultant in primary care units would facilitate identification and referral of inflammatory conditions.

Conclusion: Portuguese FP reported significant delay in hospital consulta tion after referral of suspicious AS cases. They apparently had good knowledge of AS, though responses may have been influenced by a younger, more updated and willing-to-participate physician population (selection and response bias)

Disclosure of Interests: None declared

DOI: 10.1136/annrheumdis-2019-eular.6335

\section{AB1195 PREGNANCIES IN AUTOIMMNUNE DISEASES: EXPERIENCE OF TWO CENTERS IN CALI, COLOMBIA: 2011- 2018}

Álvaro Arbeláez-Cortés ${ }^{1,2,3,4}$, Maria Andrea Moreno Salamanca ${ }^{4}$, Diana Carolina Quintero González ${ }^{3} .{ }^{1}$ Clínica de Artritis Temprana, Cali, Colombia; ${ }^{2}$ Centro Médico Imbanaco de Cali S.A., Cali, Colombia; ${ }^{3}$ Universidad Libre de Cali, Internal Medicine, Cali, Colombia; ${ }^{4}$ Universidad Libre de Cali, Cali, Colombia

Background: The outcome in pregnancy varies according to the rheu matic disease.

Objectives: To describe the pregnancies outcomes of women with rheu matic diseases at two reference centers in Cali, Colombia

Methods: Descriptive study. Records of pregnant patients attended from August 2011 to December 2018 were reviewed. Thirty-nine patients were found, 11 without a defined rheumatic entity (10 with positive ANA only, 